\title{
A elaboração de evoluções de enfermagem e possíveis dificuldades: percepção do enfermeiro
}

RESUMO | Estudo descritivo e qualitativo, com o objetivo de analisar a percepção dos enfermeiros de um hospital público do município de Belém, acerca de possíveis dificuldades, para elaboração de evoluções escritas. O estudo foi realizado no mês de setembro de 2016, nas clínicas cirúrgica e no $2^{\circ}$ Departamento de Câncer, de um hospital-escola, referência em oncologia, localizado no município de Belém/PA. Os participantes do estudo foram oito enfermeiras, entrevistadas a partir de um roteiro semiestruturado. A análise dos dados se deu através da análise de conteúdo, emergindo três categorias temáticas. As dificuldades concentram-se, principalmente, na declaração da falta de tempo, potencialmente decorrente da realização de outras atividades além das de cuidado, e agravada pelo reduzido número de profissionais, o que pode refletir na perda de informações sobre os resultados dos cuidados prestados e da avaliação de sua qualidade, e priorização de atividades administrativas. Conclui-se que, de acordo com os participantes do estudo, a falta de tempo é o principal fator dificultador para a realização da evolução de enfermagem em todos os aspectos que deve contemplar.

Palavras-chaves: enfermagem; registros de enfermagem; auditoria de enfermagem; processo de enfermagem.

ABSTRACT | Descritive and qualitative research, with the objetive to analyse the nurses perception of a public hospital in the city of Belém about the possible difficulties to elaborate written evolutions. The study was performed in September 2016, in the surgical clinics and in the 2nd Cancer Department, of a school hospital, reference in oncology, located in Belém/PA. The research's participants were eight nurses, interviewed from a semi-structured script. Data analysis was made through content analysis, originating three tematic categories. The difficulties concentrate, mainly, in alegation of lack of time, which is potentially due to activities other than care, and aggravated by the reduced number of professionals, which may reflect the loss of information about the care outcomes and the evaluation of its quality, and prioritization of administrative activities. It is concluded that, according to the participants of the study, the lack of time is the main obstacle to the achievement of the nursing record in all aspects that should contemplate.

Keywords: nursing; nursing records; nursind audit; nursing process.

RESUMEN | Estudio descriptivo y cualitativo, con el objetivo de analizar la percepción de los enfermeros de un hospital público del municipio de Belém, acerca de posibles dificultades, para la elaboración de evoluciones escritas. El estudio fue realizado en el mes de septiembre de 2016, en las clínicas quirúrgicas y en el $2^{\circ}$ Departamento de Cáncer, de un hospital-escuela, referencia en oncología, localizado en el municipio de Belém / PA. Los participantes del estúdio fueron ocho enfermeras, entrevistadas a partir de un itinerario semiestructurado. El análisis de los datos se dio a través del análisis de contenido, emergiendo tres categorías temáticas. Las dificultades se concentran principalmente en la declaración de la falta de tiempo, potencialmente derivada de la realización de otras actividades además de las de cuidado, y agravada por el reducido número de profesionales, lo que puede reflejar en la pérdida de informaciones sobre los resultados de los cuidados prestados y de la evaluación de su calidad, y priorización de actividades administrativas. Se concluye que, de acuerdo con los participantes del estudio, la falta de tiempo es el principal factor dificultador para la realización de la evolución de enfermería en todos los aspectos que debe contemplar.

Palabras claves: enfermería; registros de enfermería; auditoría de enfermería; proceso de enfermería.

\section{Ana Gracinda Ignácio Silva}

Docente do Centro Universitário Metropolitano da Amazônia. Professora adjunta aposentada da Universidade do Estado do Pará e Universidade Federal do Pará. Doutora em enfermagem pela escola de Enfermagem Anna Nery (UFRJ).

\section{Bruna Rafaela Leite Dias}

Pós-graduanda (Modalidade Residência) em Enfermagem Oncológica. Enfermeira graduada pela Universidade do Estado do Pará (UEPA).

\section{Maridalva Ramos Leite}

Docente da Universidade do Estado do Pará (UEPA). Mestre em motricidade Humana pela UEPA.

Recebido em: 01/02/2019

Aprovado em: 13/05/2019
INTRODUÇãO

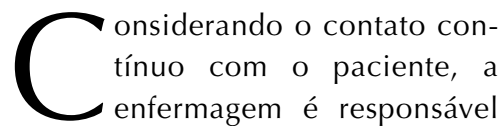
pela maioria das informações referentes ao cuidado, sinalizando aos demais membros da equipe o quadro em que se encontra o paciente, através do monitoramento de sinais vitais, sintomas e queixas. Desse modo, espera-se que os registros realizados por esta categoria permitam a comunicação permanente entre os membros da equipe multiprofissional, com transmissão de informações que facilitem o planeja- 
mento, tomada de decisões clínicas e gerenciais e continuidade da assistência prestada ${ }^{3}$.

Destaca-se, então, a evolução de enfermagem, também chamada de avaliação de enfermagem. Esta consiste no registro feito pelo enfermeiro após avaliação do estado geral do paciente com informações fidedignas do estado de saúde do enfermo, como também serve de meio de comunicação entre a equipe multiprofissional de saúde, garantindo a continuidade da assistência. Constitui-se ainda em um instrumento indispensável para a consolidação da Sistematização da Assistência de Enfermagem (SAE), que organiza o cuidado oferecido pela equipe de enfermagem e controla resultados ${ }^{4-6}$.

Uma evolução de enfermagem insuficiente, ou deficiente, contribui para perdas significativas, tanto de caráter financeiro quando do repasse de verbas, quanto de qualidade da assistência prestada ao paciente. Há comprometimento da continuidade do cuidado ao paciente, além da dificuldade para mensurar resultados assistenciais advindos da prática do enfermeiro ${ }^{7}$.

Essa problemática pôde ser observada na trajetória acadêmica, em prática curricular nas clínicas cirúrgica, ginecológica, torácica, urologia e mastologia, em Hospital Público do Estado do Pará, referência em tratamento oncológico. Consultando o prontuário do paciente, especificamente às evoluções de enfermagem, objetivando conhecer os pacientes, observamos a presença de abreviaturas não preconizadas, diagnósticos de enfermagem incompletos ou ausentes, grau de dependência do paciente não identificado, evoluções de enfermagem inelegíveis ou ausentes, o que dificultou qualquer contribuição para a aprendizagem e assistência ao cliente.

Sendo assim, considerando que as evoluções de enfermagem têm o poder de consolidar o processo de enfermagem, garantindo uma melhor comuni- cação entre a equipe de saúde e, por conseguinte, continuidade da assistência, por constituírem-se ainda como ferramentas ético-legais do cuidado prestado, dá-se a relevância da temática, surgindo o interesse em desenvolver um estudo tendo como questão norteadora: Na percepção dos enfermeiros, que fatores dificultam a elaboração de evoluções de enfermagem de forma completa? O presente estudo tem como objetivo analisar a percepção dos enfermeiros de um hospital público do município de Belém, acerca de possíveis dificuldades, para elaboração de evoluções escritas.

\section{MÉTODO}

Estudo descritivo, transversal, com abordagem qualitativa, realizado nas dependências da clínica cirúrgica e do $2^{\circ}$ Departamento de Câncer (DC), de um hospital público do Estado do Pará, localizado no município de Belém.

Os dados foram coletados no mês de setembro de 2016, através de entrevista semiestruturada, durante todos os dias da semana, com os enfermeiros de todos os turnos de trabalho, sendo os participantes selecionados a partir da técnica de amostragem não probabilística por censo. Foram participantes do estudo oito enfermeiros que atuavam nas referidas clínicas, nos três turnos: manhã, tarde e noite, e que atenderam aos critérios de inclusão do estudo: estar em atividade em uma das clínicas; aceitar participar do estudo após conhecer seus objetivos; e assinar o Termo de Consentimento Livre e Esclarecido.

As entrevistas foram individuais, compostas pela caracterização do participante e cinco questões abertas, sendo elas: "Você faz evolução de enfermagem de todos os pacientes? Em que momento?"; "Como você elabora a evolução de enfermagem? Você segue algum método?"; "O que é uma evolução de enfermagem completa no seu entendimento?"; "Que fatores/aspectos, no seu entendimento, se constituem em dificuldades para fazer a evolução de enfermagem de forma completa?"; "O que você acha que não deve faltar na evolução de enfermagem?".

A análise de dados iniciou após cada entrevista, com gravação das falas transcritas na íntegra. Posteriormente, cada transcrição foi validada por quem as concedeu e analisada tematicamente a partir de unidades de significado e respostas dos participantes, e sistematizadas em quadros, servindo de base para a análise de conteúdo ${ }^{8}$.

A proposta de estudo foi aprovada pelos Comitês de Ética em Pesquisa da Escola de Enfermagem Magalhães Barata, CAAE 56038716.6.0000.5170 e parecer $\mathrm{n}^{\circ}$ 1.596.530, e do Hospital Ofir Loyola, CAAE 56038716.6.3001.5550 e parecer $\mathrm{n}^{0}$ 1.710.051. Após aprovação, o estudo foi conduzido conforme a Resolução CNS n ${ }^{\circ} 466 / 2012,{ }^{9}$ obedecendo aos padrões éticos, assinatura do termo de consentimento livre e esclarecido pelos participantes, cujo anonimato foi mantido por identificação em codificações.

\section{RESULTADOS}

A caracterização dos participantes do estudo apresenta-se distribuída no quadro a seguir.

Os enfermeiros da pesquisa são, em sua totalidade, do gênero feminino, adultos jovens entre 20 e 50 anos, casados, com formação profissional acima de seis anos.

A partir das falas dos participantes, emergiram três categorias temáticas: Realização da evolução de enfermagem no processo de cuidar; Evolução de Enfermagem completa; Aspectos que dificultam a elaboração da evolução de enfermagem de forma completa no processo de cuidar.

Tema 1: Realização da evolução de enfermagem no processo de cuidar

Constatou-se que a elaboração das evoluções de enfermagem pelas en- 


\section{Quadro 1 - Caracterização dos participantes do estudo segundo sexo, idade, estado civil e tempo de formação, Belém-PA, 2016.}

\begin{tabular}{lllll} 
Participante & Sexo & Idade (Anos) & Estado Civil & Tempo de formação (Anos) \\
E1 & F & $20-30$ & Solteiro & $0-5$ \\
E2 & F & $31-40$ & Casado & $0-5$ \\
E3 & F & $20-30$ & Solteiro & $6-10$ \\
E4 & F & $31-40$ & Casado & $6-10$ \\
E5 & F & $31-40$ & Casado & $6-10$ \\
E6 & F & $41-50$ & Casado & + de 10 \\
E7 & F & $41-50$ & Divorciado & + de 10 \\
\hline E8 & F & $41-50$ & Casado & + de 10 \\
\hline
\end{tabular}

Fonte: estudo descritivo, Hospital Ofir Loyola, 2016.

fermeiras pesquisadas no processo de cuidar tem suas características individuais e comuns. São realizadas em determinado tempo com alguns critérios, como ser dividida durante o plantão dos enfermeiros do turno diurno. São realizadas na admissão dos pacientes; no pré e pós-operatório e de acordo com a gravidade dos mesmos. No noturno, as evoluções ocorrem na admissão dos pacientes, intercorrências, em pós-operatório imediato, retorno de CTI e óbito.

Verificou-se pontos comuns, inclusive em evoluções de pacientes em estado crítico, como a unanimidade da utilização do exame físico céfalo-caudal, nível de consciência, orientação, queixas principais, sinais vitais, hidratação, sinais e sintomas, eliminações, identificação de necessidades básicas afetadas e condutas tomadas.

O discurso sobre a construção da evolução de enfermagem aponta a mesma como uma receita de bolo e o exame físico céfalo-caudal uma alternativa para abordar todos os sistemas do corpo humano, sem deixar de registrar algo por falha na memória. Há também a preocupação em comparar registros dos profissionais e plantões, para avaliar se houve mudança de estado de saúde e veracidade de informações.

Tema 2: A evolução de enfermagem completa

As enfermeiras acreditam que a evolução de enfermagem completa, consiste naquela realizada na admissão do paciente, quando na leitura se torna possível a visualização do quadro clínico do paciente, incluindo dados sociais, início do adoecimento, diagnóstico da doença, diagnóstico médico, intercorrências e descrição das condutas tomadas com avaliação do resultado. Deve ser concisa de fácil entendimento, organizada, legível, aplicação correta de termos técnicos e conhecimento técnico-científico, além de constar o julgamento clínico do enfermeiro sobre a evolução clínica do paciente.

Tema 3: Aspectos que dificultam a elaboração da evolução de enfermagem de forma completa no processo de cuidar

As participantes percebem unanimemente, a falta de tempo como principal fator que impossibilita a não realização da evolução de enfermagem completa. E, possível excesso de atribuições do enfermeiro, informações repassadas pelos pacientes, não seguir ordem cronológica e sequencial para elaboração da evolução de enfermagem, termos técnicos incorretos, erros ortográficos, falta de assinatura, carimbo e letra incompreensível.

\section{DISCUSSÃO}

A primeira categoria aborda sobre a realização da evolução de enfermagem no processo de cuidar. Este, por sua vez, se assemelha como premissa essencial da enfermagem, como tentativa de evitar o sofrimento alheio, ou ao identificar o sofrimento de um indivíduo, realiza medidas para erradicar ou reduzir o sofrimento ${ }^{10}$.

Evidencia-se que os enfermeiros elaboram seus registros sobre os pacientes de acordo com uma literatura específica, que orienta o exame físico no sentido céfalo-caudal, com descrição de necessidades ${ }^{11}$.

Referem que, o estado de saúde do paciente deve constar na evolução de enfermagem de forma compreensiva, com clareza, com informações essenciais, disposto de forma legível, colaboram com o princípio de que, sem esses procedimentos, pode ocorrer prejuízo ao paciente pelo simples fato de uma escrita manual e ilegível desestimular a leitura e compreensão do prontuário por outros profissionais ${ }^{12}$.

Acredita-se na viabilidade dos registros de enfermagem como instrumento de organização do trabalho de enfermagem, inclusive na documentação das etapas da SAE, então, é necessário que as enfermeiras comunguem desta afirmativa, para, então, torná-la concreta na prática assistencial ${ }^{13}$.

A segunda categoria retrata a com- 
preensão dessas enfermeiras quanto à evolução de enfermagem completa. Pauta-se na organização do texto para que na leitura, visualize-se o estado de saúde do paciente e diagnóstico médico. Neste sentido, entende-se que a comunicação entre os profissionais de saúde é fundamental para a organização e o (re) planejamento do processo do cuidado humano, pois registros adequados objetivam a continuidade da assistência prestada e permitem fomentar pesquisas e auditorias, a fim de contribuir para uma enfermagem mais científica $^{12}$.

No terceiro tema, visualiza-se os obstáculos para a elaboração da evolução de enfermagem completa, o tempo e a realização de outras atribuições foram apontados como principais barreiras. Esse resultado confirma estudos que apontam a falta de tempo relacionada com o excesso de atividades do enfermeiro, a prática de enfermagem se distanciando do objetivo principal: o cuidado ao paciente ${ }^{14}$.

Em muitas situações, o cumprimen- to de atividades administrativas e burocráticas é exigido pela própria instituição de saúde, visto que o mercado de trabalho espera que a enfermeira realize o controle administrativo da instituição ${ }^{15}$.

Quanto à comunicação deficiente do paciente relacionada a informações sobre sua saúde, a instituição onde se deu a pesquisa é referência estadual para o tratamento oncológico, e recebe pacientes da capital e zona rural, o que talvez dificulte o entendimento entre paciente e profissional. Nesse caso, é importante enfatizar a importância de teorias de enfermagem, como a Teoria Transcultural de Madeleine Leininger, que orienta o enfermeiro no estabelecimento de comunicação considerando as diferenças de crenças, valores, experiências e linguagem ${ }^{16}$. Quanto aos registros incorretos, incompreensíveis e incompletos relatados, é importante enfatizar que a credibilidade profissional da enfermagem implica em registros técnicos, informação substancial, consistente, clara e objetiva, data, hora, letra legível, assinatura e inscrição no COREN de sua jurisdição ${ }^{17}$.

\section{CONCLUSÃO}

O estudo permitiu evidenciar que, de acordo com os participantes do estudo, a falta de tempo é o principal fator dificultador para a realização da evolução de enfermagem de forma completa. Entretanto, existem outros fatores como o excesso de atribuições do enfermeiro, informações deficientes repassadas pelos pacientes, não utilização correta dos termos técnicos, erros ortográficos, falta de assinatura e carimbo e letra inelegível, o que pode refletir na perda de informações sobre os resultados dos cuidados prestados e da avaliação de sua qualidade.

Ante o exposto, acredita-se que este estudo contribui para a reflexão acerca da importância das evoluções de enfermagem e da implantação da SAE de forma crítica, em todas as instituições de assistência em saúde, tanto para a valorização da enfermagem quanto para dar segurança ao paciente.

\section{Referências}

1. Pedrosa KKAP, Souza MFG, Monteiro Al. 0 enfermeiro e o registro de enfermagem em um hospital público de ensino. Rev. RENE. 2011 Jul./Set.; 12(3):568-573. 2. Françolin L, Brito MFP, Gabriel CS, Monteiro TM, Bernardes A. A qualidade dos registros de enfermagem em prontuários de pacientes hospitalizados. Rev Enferm UERJ. 2012 Jan./Mar; 20(1):79-83.

3. Barroso TS. A importância do relato na evolução de enfermagem para a auditoria. Acta Biomed. Bras. 2016 Dez.; 7(2):39-49.

4. Conselho Federal de Enfermagem. Resolução n 358, de 15 de outubro de 2009. Dispõe sobre a Sistematização da Assistência de Enfermagem e a implementação do Processo de Enfermagem em ambientes, públicos ou privados, em que ocorre o cuidado profissional de enfermagem e dá outras providências [internet]. Diário Oficial da União. 2009 out. 23 [citado 2019 jan 29]. Disponivel em: <https://enfermagem. jatai.ufg.br/up/194/o/Resolu\%C3\%A7\%C3\%A3o_n\%C2\%BA358-2009.pdf>.

5. Papeschi MSS, Toledo Neto JL, Katakura EAL, Melo SCCS, Silva NMMG. A importância das anotações de enfermagem na auditoria hospitalar. Rev Odontol (ATO). 2014 Mai.; $14(5): 308-324$.

6. Soares MI, Resck ZMR, Terra FS, Camelo SHH. Sistematização da assistência de enfermagem: facilidades e desafios do enfermeiro na gerência da assistência. Esc. Anna Nery Rev. Enferm. 2015 Jan./Mar.; 19(1):47-53.

7. Camargo LRL, Pereira GR. Análise dos registros realizados pela enfermagem e o possível impacto na auditoria: uma revisão da literatura nacional. Rev. Adm. Saúde. 2017 Jul./Set.; 17(68):1-11.

8. Bardin L. Análise de conteúdo: edição revista e ampliada. São Paulo: Edições 70; 2016.

9. Conselho Nacional de Saúde. Resolução n 466, de 12 de dezembro de 2012 [internet]. Diário Oficial da União. 2012 Dez. 12 [citado 2019 mai. 02]. Disponível em: <http://bvsms.saude.gov.br/bvs/saudelegis/cns/2013/res0466_12_12_2012.html>.
10. Beserra EP, Oliveira FC, Ramos IC, Moreira RVO, Alves MDS, Braga VAB. Sofrimento humano e cuidado de enfermagem: múltiplas visões. Esc. Anna Nery Rev. Enferm. 2014 Jan./Mar.; 18(1):175-180.

11. Universidade Federal do Paraná. Hospital de Clínicas. Diretoria de Enfermagem - Comissão de Sistematização da Assistência de Enfermagem (COMISAE). Avaliação de Enfermagem: anamnese e exame físico (adulto, criança e gestante) [internet]. Curitiba: UFPR; 2014 [citado 2019 jan 29]. Disponível em: <http://www2.ebserh.gov. br/documents/1948338/2326847/livreto_sae\%5B1\%5D.pdf/d4fa3a69-f9f2-41f1-9e89-f49010ca27bc>.

12. Seignemartin BA, Jesus LR, Vergílio MSTG, Silva EM. Avaliação da qualidade das anotações de enfermagem no pronto atendimento de um hospital escola. Rev. RENE. 2013 Jun./Nov.; 14(6):1123-1132.

13. Araujo MM, Diniz SOS, Silva PS. Registros de enfermagem: reflexões sobre o cotidiano do cuidar. ABCS Health Sci. 2017; 42(3):161-165.

14. Moraes AT. 0 cotidiano do cuidar de enfermeiros em um hospital da Zona da Mata Mineira: compreendendo o dia a dia do fazer enfermagem [dissertação]. Juiz de Fora: Universidade Federal de Juiz de Fora; 2014.

15. Gelbcke FL, Relbnitz KS, Prado ML, Lima MM, Kloh D. A práxis da enfermeira e a integralidade do cuidado. Enferm. foco. 2011 Mar./Abr; 2(2):116-119.

16. Seima MD, Michel T, Méier MJ, Wall ML, Lenardt MH. A produção científica da enfermagem e a utilização da teoria de Madeleine Leininger: revisão integrativa 1985 -2011. Esc. Anna Nery Rev. Enferm. 2011 Out/Dez.; 15(4):851-857.

17. Conselho Federal de Enfermagem. Câmara Técnica de Legislação e Normas. Guia de recomendações para registro de enfermagem no prontuário do paciente e outros documentos de enfermagem. Brasilia: COFEN; 2016 [citado 2019 jan 29]. Disponível em: <http://www.cofen.gov.br/wp-content/uploads/2016/08/Guia-de-Recomenda\%C3\%A7\%C3\%B5es-CTLN-Vers\%C3\%A3o-Web.pdf>. 